\title{
A generalized inhomogeneous Markov chain occupancy model for open-plan offices using Real Time Locating System data
}

\author{
Shide Salimi, Amin Hammad \\ Concordia University, Montreal, Canada
}

\begin{abstract}
A good occupancy prediction model requires enough input data pertinent to the occupants' space utilization patterns. However, most of the occupancy detection systems cannot provide this detailed information, which reduces their practicality for open-plan offices. Therefore, there is a need to use proper sensing techniques to distinguish between different occupants in open-plan offices when detecting occupancy patterns. In this study, the probabilistic occupancy modelling has been further enhanced using inhomogeneous Markov chain prediction model based on data collected by a Real Time Locating System (RTLS). The comparison between the occupancy profiles resulting from the prediction model and the actual profiles showed that the prediction model was able to capture the behaviour of occupants. An adaptive probabilistic occupancy prediction model, which distinguishes the temporal behaviour of different occupants within an open-plan office, allows for the application of occupancy-centred local control strategies.
\end{abstract}

\section{Introduction}

Buildings are responsible for $41 \%$ of the total energy consumption in the United States $(22 \%$ for residential buildings and 19\% for commercial buildings), 31\% in Canada (17\% for residential buildings and $14 \%$ for commercial buildings), and $41 \%$ in Europe (27\% for residential buildings and $14 \%$ for commercial buildings) (US Energy Information Administration (EIA), 2013; BPIE Database, 2013; Hong, et al., 2015; Natural Resources Canada, 2015). Therefore, the intelligent use of energy within buildings is a recent trend of research studies and is the target of Building Energy and Comfort Management systems (Nguyen and Aiello, 2013).

According to the International Energy Agency (IEA), Energy in the Buildings and Communities Program (EBC), Annex 53 the following parameters are the most influential for energy consumption in buildings: (1) climate, (2) building envelope, (3) building energy and service systems, (4) indoor design criteria, (5) building operation and maintenance, and (6) occupant behaviour (Annex 53, 2016). Some of these parameters are easy to determine, being related to the physical characteristics of the building (e.g., building size, orientation, construction materials, Heating, Ventilation, and Air Conditioning (HVAC) system size and type, etc.). On the other hand, some parameters that vary with time are difficult to predict, such as occupancy input. Thus, providing a comprehensive and reliable occupancy model is still under development.

Precise estimation of buildings' energy consumption requires reliable occupancy models that can generate detailed occupancy information with acceptable level of accuracy. Having proper resolution level of data regarding space utilization patterns is a key factor for developing accurate occupancy models. Occupancy data are highly context dependent and they can provide answers to the following questions: (1) How many people are present in a space? (2) Who are they? (3) For how long are they present in the space? and (4) What are they doing? Having this information helps to determine the occupants' interactions with building systems (Hong, et al., 2015). This will eventually lead to the application of occupancy-centred local control strategies on the building systems. Furthermore, occupancy-related information is useful for different energy management purposes as well as other areas, such as safety, security, and emergency response. According to the research results and industrial practices, considering occupancy model by collecting real-time data to create occupants' profiles of energy usage patterns results in up to $88 \%$ savings in energy consumption (Ardakanian, et al., 2016; Wang, et al., 2017; Fauzi \& Sulistyo, 2018).

The above discussion shows the importance of capturing detailed-enough occupancy data and the usage of occupant behaviour analytics (data processing) steps to filter the input data and create a reliable occupancy model. Monitoring and data collection are important steps to capture the occupants' space utilization patterns. This data is gathered for a reasonable period through monitoring techniques, such as Real Time Locating Systems (RTLSs). However, most of the occupancy detection systems cannot provide the number of occupants and their specific locations (i.e., the $x$ and $y$ coordinates of the occupants) when they are used for open-plan offices. Most of the research works that considered shared multi-occupied offices did not distinguish between different individuals. Therefore, their practicality is reduced for open-plan offices, which have multiple thermal zones (Li, et al., 2012). Therefore, there is a need to use proper sensing and occupancy modelling techniques to distinguish the temporal behaviour of different occupants within an open-plan office.

This paper aims to develop a new adaptive probabilistic occupancy prediction model for open-plan offices based 
on occupancy data. In this study, the occupancy modelling (i.e., occupants' profiles) has been further enhanced using inhomogeneous Markov chain prediction model, which distinguishes the temporal behaviour of different occupants within an open-plan office based on occupancy space utilization patterns data. To this end, the occupants' detailed data (who, where, when) are collected using a relatively new monitoring technology (i.e., Bluetooth RTLS) that responds to occupancy changes in open-plan office buildings with acceptable accuracy. After developing the personal profile for each occupant with varying time-steps using advanced data analytics, a new adaptive probabilistic occupancy prediction model is developed to be used for occupancy prediction of openplan offices. The proposed model is verified using a case study. Finally, comparing the building's real occupancy and the results of the prediction model provides the validation of the applicability of the proposed model.

\section{Literature Review}

A comprehensive literature review with the focus on different techniques used to monitor and collect occupancy data, as well as occupancy modelling in office buildings has been conducted by the authors (Salimi and Hammad, 2018). In recent years, radio frequency (RF)based localization technologies, such as $\mathrm{Wi}-\mathrm{Fi}$ and Bluetooth are widely used by researchers due to their deployment flexibility, communication range and ability to work without line of sight. The ability to track multiple moving objects in real-time makes Bluetooth Low Energy (BLE) systems an optimal choice for different applications, such as building energy efficiency, sport, and healthcare applications, optimizing store layout, security, and emergency situations (Quuppa, 2017; Salimi and Hammad, 2018).

Comparing different research works showed that detailed data regarding occupants' location, their number, identities, and activities can be collected using BLE location sensors (Salimi and Hammad, 2018). Different occupants in open-plan offices can be temporally distinguished using high-resolution occupancy data, which eventually results in a reliable and accurate estimation of occupants' space utilization patterns.

Leveraging stochastic modeling of occupants' parameters and profiles helps to reflect the dependencies of occupancy models on the season, weather, time of day, and occupant habits and personality (Haldi and Robinson, 2008; Chang and Hong, 2013). Stochastic methods use real data pertinent to the occupants' location, movement, and actions, being collected over a reasonable period, to predict the probability of an event (i.e., occupant being present in a space) or an activity (e.g., window opening behaviour) and generate the probabilistic profiles (Virote and Neves-Silva, 2012; Wei, et al., 2018). Monte Carlo methods, Markov Chain, discrete and semi-hidden Markov Chain models, as well as Poisson model are in the category of stochastic methods. Since the occupant's next state is highly dependent on his/her present state, which is the basis of the Markov chain process, modelling occupancy space utilization patterns using Markov chain is one of the most utilized techniques by researchers (Salimi and Hammad, 2018).

This study is compared with different research works that used Markov chain models in terms of the type of Markov chain model, occupancy model level, type of space, and information resolution derived from the occupancy model. Although some similarity can be seen between the reviewed papers and the current paper, none of them developed the occupancy model at individual, zone, and room levels. In addition, the proposed occupancy prediction model is a generic model that can be used for offices with any size and any number of occupants (i.e., shared and private). This makes the proposed model be independent of the size of the monitored area as well as the number of occupants. Moreover, the proposed occupancy model provides high-resolution information regarding variations in occupancy patterns by considering the temporal behavior of occupants through defining the detailed work states, which are explained in detail in the next section. Finally, none of the reviewed papers considered the combination of all these aspects in a realworld scenario. More details regarding the occupancy monitoring and modelling can be found in Salimi and Hammad (2018).

\section{Research Method}

The occupants' presence data, including their identities, durations of presence, and locations in different zones of a building, determine the accuracy of the occupancy prediction model. After collecting this data using the BLE RLTS, the occupancy prediction model is used to determine the occupants-specific probabilistic profiles based on their presence data.

In order to capture detailed occupancy information in real open-plan offices and improve the accuracy of the occupancy prediction model, open-plan offices should be divided into multiple zones. The zoning concept enables the occupancy prediction model to assign different probabilistic occupancy information to each zone. The zoning is applied to consider the effect of (1) different types of activities performed in each zone; (2) different number of the HVAC terminal units or the number of luminaires; and (3) different facade orientation for perimeter zones, to name a few.

To consider the variations in the occupants' profiles due to their temporal behaviour, each day is divided into different time slots. There are typical events of importance in office buildings that should be captured while defining these time slots, such as the first arrival to the office. These time slots are determined based on the patterns seen in the collected data as will be explained in the following sections. The events of importance indicate the typical patterns of the occupants' activities in openplan offices. These activities are referred to as work states in this study as shown in Table 1 . The duration of each work state is determined using the monitoring data. The first arrival to the office is defined as the first reading of the occupant's presence in the office after his/her long absence during the night. The last departure from the office is determined as the point when there is no 
recording of the occupants' presence for a duration greater than four hours after that point. Lunch break is defined as a break happening around noon with the duration greater than half an hour. Other breaks during the day with duration shorter than half an hour are considered as short breaks. Meetings, as one example of long breaks, are events that are happening based on a predefined schedule, such as weekly, bi-weekly, etc.

Table 1: Typical occupancy work states in office buildings.

\begin{tabular}{clc}
\hline $\begin{array}{c}\text { Work } \\
\text { State }\end{array}$ & Description & $\begin{array}{c}\text { Label (in } \\
\text { Prediction Model) }\end{array}$ \\
\hline 1 & $\begin{array}{l}\text { Working in Occupant's Station } \\
\text { (Occupant's Zone) }\end{array}$ & $\mathrm{S}_{\mathrm{oc}}$ \\
\hline 2 & $\begin{array}{l}\text { Working in Other Occupants' } \\
\text { Station (Other Zones) }\end{array}$ & $\mathrm{S}_{\mathrm{ot}}$ \\
\hline 3 & Lunch Break (L) & $\mathrm{S}_{\mathrm{lb}}$ \\
\hline 4 & Short Break (SB) & $\mathrm{S}_{\mathrm{sb}}$ \\
\hline 5 & Long Break/Meeting (LB) & $\mathrm{S}_{\mathrm{lm}}$ \\
\hline
\end{tabular}

The proposed framework comprises of three main steps including data collection, data processing, and occupancy prediction model as shown in Figure 1

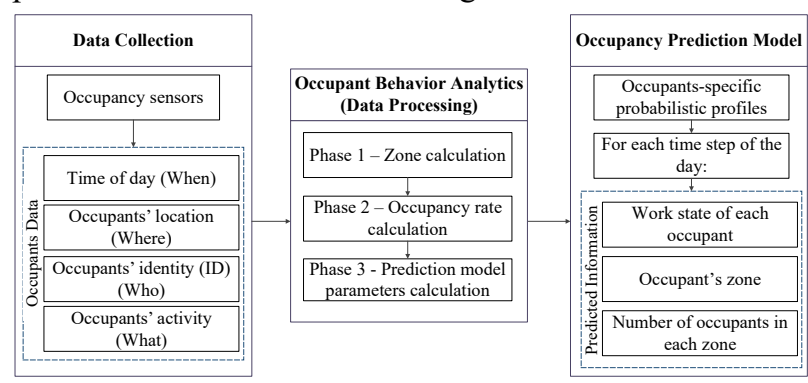

Figure 1: Proposed framework for the probabilistic occupancy prediction model.

As mentioned in the Introduction section, occupants should be monitored over a reasonable period using RTLS to get occupants' location, their identities, presence time, and the type of activities and find the spatiotemporal patterns of the occupants' behaviour. To do so, either tags or cell phones can be used to send signals to the RTLS. The monitoring system then localizes occupants within a space using the collected data and gathers the required occupancy data.

During the occupant behaviour analytics (data processing), analysis is required to find important occupancy features, such as the number of present occupants, periods of absence and presence, and other occasional variations in the occupants' profiles.

Knowing that the future state of the occupant depends on his/her current state, the Markov chain technique is used in this research to develop the probabilistic occupancy profiles. Random mobility between different work states is assumed to account for the occupants' movement among the zones inside and outside open-plan offices. This assumption allows modeling the transitions among work states in Markov matrices. Therefore, the next work state of the occupant only depends on his/her present state and some rules about work states.

At the beginning of Phase 1 (zone calculation) of data processing steps, the total daily presence time $(P T)$ of each occupant in office room is calculated from the raw data. In the next step, the zone of each occupant is calculated according to the $x$ and $y$ coordinates of his/her tag for each time-step. This procedure is repeated for each day $(d)$ of each week of the data collection period $(w)$ and for each occupant $(o)$. Therefore, the occupants' zones for each time-step of $P T$ are determined at the end of Phase 1 .

In the first step of Phase 2 (occupancy rate calculation), the total occupancy duration (TOD) of the office being occupied is calculated. To do so, the start and end times of occupancy are respectively equal to the earliest first arrival to the office and the latest departure from the office among all the occupants. The difference between the start and end times of occupancy determines the TOD. Then, the number of present occupants, and eventually the occupancy rate for time-step $t,\left(o c c_{r}^{t, d}\right)$, are determined according to Equation (1) at zone and room levels for each day of a week $(d)$ including weekends, and for the total number of weeks of the data collection period $(W)$.

$$
o c c_{r}^{t, d}=\frac{\sum_{w=1}^{W}\left(\frac{n_{o}^{t, d}}{N_{o}}\right)_{w}}{W} \times 100 \%
$$

where $n_{o}^{t, d}$ is the number of present occupants at time-step $t$ and day $d$, and $N_{o}$ is the total number of occupants sharing the same open-plan office during day $d$.

Having the occupancy rate, a regression curve is fitted to each set of data (i.e., the occupancy rate for all time-steps) to represent the changes of the occupancy rate with time for each day of a week. This information helps to calculate the internal load from the occupancy presence for the HVAC system operation at zone and room levels.

Phase 3 (prediction model parameter calculation) of the data processing procedure comprises of the analysis required to obtain the occupant-specific transition probability matrices for each time-step of each day of a week. This phase starts with changing the time-step resolution according to the purpose of the occupancy model. For instance, HVAC system local control strategies require longer time-steps compared to lighting control strategies knowing that it takes time for the system to adjust the zone temperature. After calculating the occupant's zone for the new time-step, the work state $(i)$ of the occupant is determined based on the collected data. The probability of being at work state $i(i=1,2, \ldots, I$, where $I$ represents the maximum number of work states) at each time-step $t, s_{o, i}^{t, d}$, along with the probabilities of transition occurrences between different work states are calculated over the monitoring period $\left(t r_{o, i j}^{t, d}\right)$. Next, the transition probabilities between different work states, as defined in Table 1, are calculated using $s_{o, i}^{t, d}$ and $t r_{o, i j}^{t, d}$ :

$$
\begin{aligned}
& \left.P_{i j}^{d}(t)=1-s_{o, i}^{t, d}+s_{o, i}^{t, d} \times t r_{o, i j}^{t, d} \quad \text { (if } i=j\right) \\
& P_{i j_{o}}^{d}(t)=s_{o, i}^{t, d} \times t r_{o, i j}^{t, d} \quad(\text { if } i \neq j)
\end{aligned}
$$

These formulas are inspired by the work of Yamaguchi et al. (2003). However, two improvements are applied to their proposed formula. Firstly, the Markov chain is time- 
independent in their method. Secondly, they assumed constant numbers for the parameters $s$ and $t r$. In this study, the Markov chain and the parameters are timedependent. In addition, the collected data regarding the actual occupancy of the open-plan office (on-site experiments) are used to define the parameters $s_{o, i}^{t, d}$ and $t r_{o, i j}^{t, d}$ with some enhancement in their calculation method as discussed above.

Considering five states of the transition probability matrix, this matrix has a dimension of $5 \times 5 \times 288 \times 7$ using 5-minute time-step for one day (i.e., 288) and one matrix for each day of a week (i.e., 7 days).

In the next step, Probability Density Function (PDF) for each time-step $t$ can be deduced from each row of the Markov transition matrix. Further, the Cumulative Distribution Function (CDF) is derived from the PDF for each time-step. The CDF is a histogram of five bins corresponding to the five work states. Each bin shows the probability at which a value of that bin can be randomly selected. Using the Inverse Function Method (IFM) gives the estimation of the work state for the next period $(t+$ 1 ). The IFM works by inverting the CDF of the parameter of interest. It randomly generates a number between 0 and 1 using a uniform distribution. The random number determines which bin is going to be selected for the parameter of interest using the CDF. Figure 2 illustrates these steps.

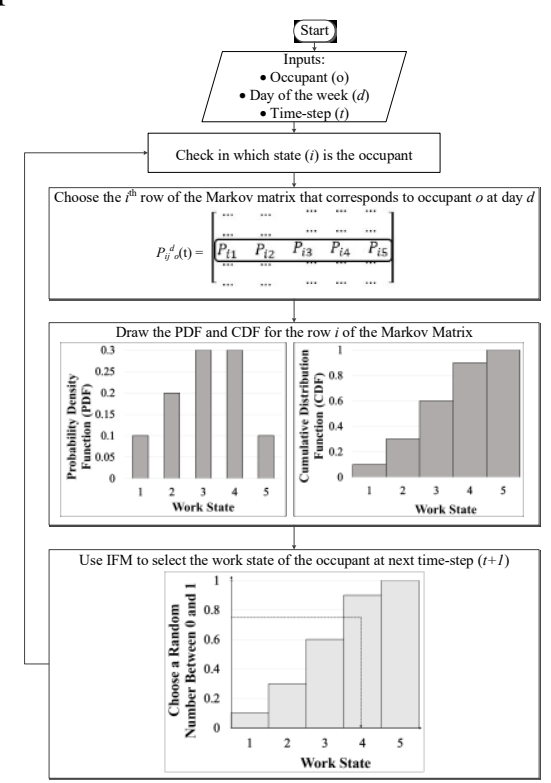

Figure 2: Work state estimation flowchart.

\section{Markov Chain Occupancy Prediction Model}

As mentioned in Research Method section, the whole day is clustered into different time slots to reflect the temporal aspect of occupants' behaviour. As a result, the probability of occurrence of different states varies with the time of the day; and consequently, the transition probability matrices are different for each of these time slots. For instance, if an occupant is going out of the office (at time $t$ ) for the lunch break at time $t+1$, there is a higher probability to either stay at lunch break or go back to his/her zone at time $t+2$ and no probability to go to a short break. This makes the transition probabilities timedependent. This type of Markov chain process is called inhomogeneous Markov chain (Douc, et al., 2004).

In the proposed inhomogeneous Markov chain model for prediction of space occupancy in multi-occupied offices, the states of the Markov chain are occupants' work states as shown in Table 1 . This results in having $5 \times 5 \times 288 \times 7$ transition probability matrices independent of the maximum number of occupants in open-plan offices. Compared to methods that define transition probability matrices based on the number of occupants in a zone, [e.g. Richardson, et al., (2008); Han, et al., (2012); Ai, et al., (2014)], or methods that consider some restrictions regarding the movement of occupants between zones to reduce the order of transition matrices [e.g. Chen, et al., (2015)], using the proposed method significantly simplifies the calculation of transition probability matrices. Transition probability matrices are key parameters in Markov chain models and reducing their order has a high impact on the overall complexity of the Markov models, especially for inhomogeneous Markov chain models with many transition matrices.

The output of the proposed inhomogeneous Markov chain model is the probabilistic profiles of each specific occupant. Important occupancy features can be derived from these profiles. Eventually, building energyconsuming systems are adjusted based on this information to reflect the variations in different occupants' daily profiles.

\section{Implementation and Case Study}

Figure 3 shows the picture of the case study location (a research laboratory). There are six occupants assigned to two zones within the research laboratory. The monitored occupants are graduate students conducting research in different levels of education including master and $\mathrm{PhD}$ students. The BLE-based monitoring system used in this research (i.e., Quuppa Intelligent Locating System ${ }^{\mathrm{TM}}$ ) can track the latest smartphones and BLE devices with the accuracy of 20-50 cm (Grizzly Analytics, 2015; Liu, 2017). The size of the monitored office is $5.0 \mathrm{~m} \times 7.0 \mathrm{~m} \times 3$ $\mathrm{m}$. In order to get the required data for the prediction model, it is important to know whether the occupant is at zone 1,2 or 0 (which is outside the office). According to the dimensions of each zone (i.e., $5.0 \mathrm{~m} \times 3.5 \mathrm{~m}$ ), the accuracy of $20-50 \mathrm{~cm}$ is precise enough for this study.

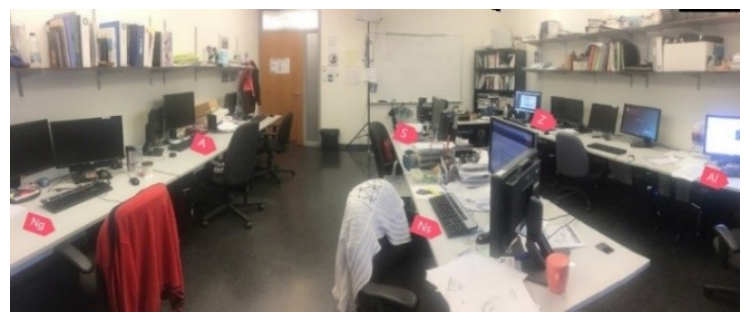

Figure 3: Case study location (graduate research lab).

In this study, the test was run for one year and since the collected data from the monitoring system could be used for different purposes with different levels of accuracy, the BLE system monitored occupants with high resolution (i.e., each second). However, the high granularity of one 
second is not required for building energy management Therefore, the occupants' zones are calculated every minute. During the one-minute time-step, the final selected zone for that time-step will be the zone in which the occupant spent more seconds.

\section{Results}

Temporal variations in occupancy presence data are shown by analysing the changes in the occupancy $P T$ for two time horizons (i.e., daily and monthly) based on the one-year collected data.

Using the data analytics steps, the $P T$ of occupants for each day of a week (weekdays and weekends) and at each zone are derived and the results are shown in Figure 4 for zone 1. For the first half of the year (i.e., the first 27 instances of days), average $P T$ is around 600 minutes for zone 1 . This number drops $33 \%$ to almost 400 minutes for the second half of the year. However, the average $P T$ of the first half of the year rarely goes under 400 minutes besides the beginning of the year that shows smaller $P T$ due to the holidays. This indicates that office occupants tend to spend more time outside of the office when the outside weather gets better during the spring and summer. Therefore, it is important to consider the seasonal variations when defining occupancy profiles. The same data is generated for zone 2 as well as for weekend for both zones, which are not included here due to space limitation. As it was expected, the occupancy rate of the office is much lower during the weekends. However, it is important to know that the office is always occupied for several hours during the weekends.

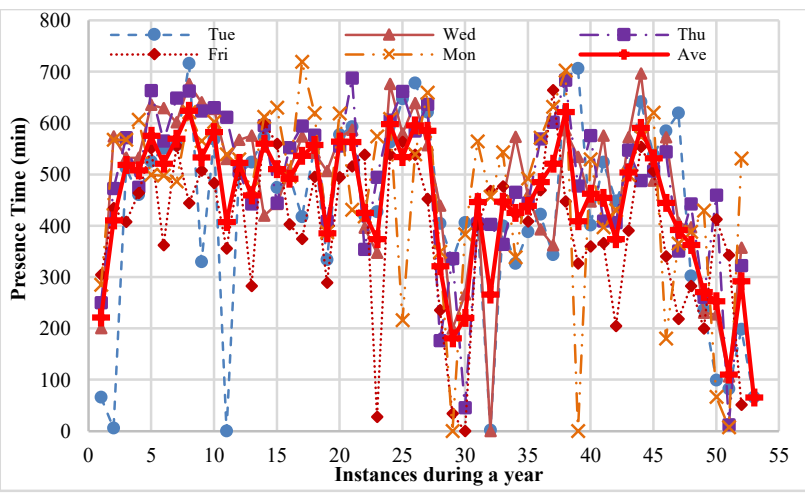

Figure 4: Variation in zone 1 occupancy PT for different days of a week and the average PT.

The occupancy $P T$ over different months of the year is illustrated in boxplots for both zones as demonstrated in Figure 5. It can be seen from this graph that the $P T$ of occupants in zone 2 on average exceeds that of zone 1 during all months of the year. The highest difference happens during the month of January in which zone 1 had lower $P T$ than zone 2 during more than $75 \%$ of the days. These boxplots also show that the $P T$ is more consistent in zone 2 and remains at upper levels while zone $1 P T$ is more variable especially at lower levels. In zone 2 , the $P T$ is higher than six hours for $75 \%$ of the days of each month except for December. The most symmetric data is in July for zone 2. Zone 2 consistent $P T$ makes predictions more dependable than the highly variable $P T$ of zone 1 . The collected data is skewed to the left for most of the first nine months of the year at zone 1. This observation is aligned with the conclusion that occupancy in zone 2 varies much less than that of zone 1 .

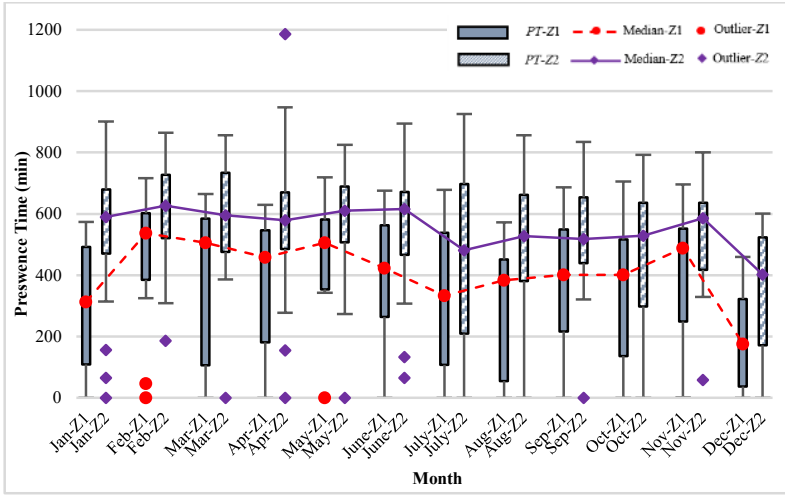

Figure 5: Variation in occupancy PT for different months of a year.

Variations in the median of $P T$ can also be observed in this graph. December shows the lowest occupancy within all months and in both zones followed by January. The median $P T$ then starts to increase and is almost stable during the next five months. Furthermore, distinguished drops can be detected during July and August. There is an increase in the $P T$ for the following months. However, the median shows lower values for the second half of the year compared to the first half at both zones. The much longer whiskers for $P T$ of zone 2 in April and August indicate that $P T$ varies more widely during these two months. These drops and variations could be due to the spring and summer break times. This is an indication of seasonal variations in the $P T$. The peak of the median occupancy is observed during February for both zones. Some outliers are observed especially at zone 2 . These abnormal values can affect the overall observation due to their very high or low extreme values, and hence should be discarded from the data series. Removing these points avoid underestimating or overestimating the $P T$ at both zones.

Overall, the above discussion shows the importance of applying the effect of temporal behaviour of occupants when predicting their presence patterns. The remaining results shown in this study are derived using one month of data collection. After determining the occupants' zones for each one-minute time-step of $P T$, the average number of occupants at each zone of the space are calculated for each day of a week within one month of the data collection. A regression curve is fitted to each data set (i.e., the average occupancy rate for each day of a week) to represent the changes of the occupancy rate with time. Figure 6 shows the fitted curve for Mondays.

\section{Validation of the Occupant Behaviour Analytics Method}

In order to process the raw data to reflect the five work states, the ranges derived from ground truth data, as a basis for data processing, are broken down to smaller time slots as demonstrated in Figure 7. To do so, the CDF of the events of importance, such as the first arrival to the office, are created to determine the actual range of their occurrences. For instance, the majority of the first arrival 
events have occurred between $08: 15$ to $11: 30$ am as shown in this figure.

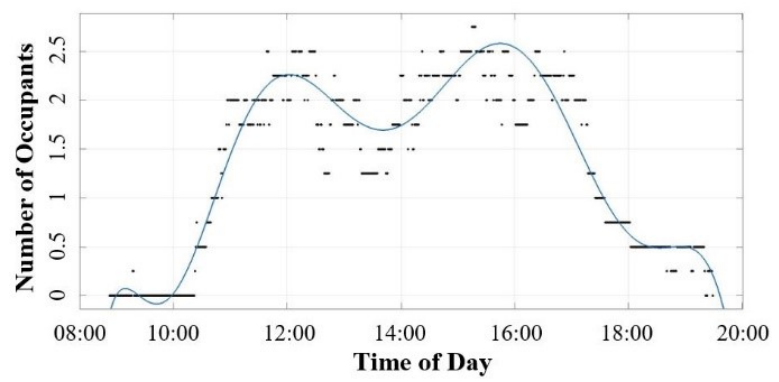

Figure 6: Fitted curve on the collected data for Mondays.

Comparing the obtained daily occupancy profiles with those obtained from one-month ground truth data showed some differences that are found between the arrival times and the results of the occupant behaviour analytics method. The review of the raw data in these cases shows that the occupants arrived and left the room after a short stay (less than five minutes). This results in delay for the processing methods to capture the first arrival of the occupants. For the other events of importance, the occupant behaviour analytics method could catch them with high accuracy. Thus, it can be concluded that the daily profiles are in accordance with the ground truth data.

\section{Occupancy Prediction Results using Proposed Model}

To estimate the occupancy profiles using inhomogeneous Markov chain occupancy prediction model, all states are labelled to show the transition probabilities from one state to another according to the last column of Table 1 .

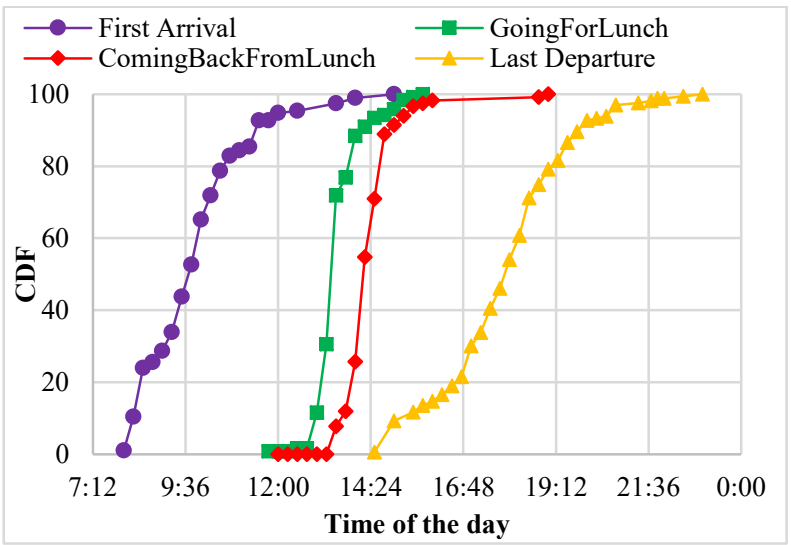

Figure 7: Cumulative distribution functions of events of importance during a day at office level.

Different resolution levels are required to control different building systems according to the discussion in the Research Method section. To reflect on this logic, fiveand 30-minute prediction time-steps are considered to determine occupancy predictions for lighting and HVAC systems control, respectively. As mentioned before, six probability transition matrices with dimension of $5 \times 5 \times 288 \times 7$ are generated for six occupants of the office. For instance, the transition matrix of occupant $O_{2}$ on Mondays at 05:45 pm is shown below:

$$
\begin{aligned}
& P_{i j}{ }_{o_{2}}^{\text {Mon }}(05: 45 \mathrm{pm}) \\
& =\left[\begin{array}{ccccc}
0.7778 & 0.1111 & 0 & 0 & 0.1111 \\
0.1111 & 0.7778 & 0 & 0 & 0.1111 \\
0 & 0 & 0 & 0 & 0 \\
0 & 0 & 0 & 0 & 0 \\
0.1111 & 0.1111 & 0 & 0 & 0.7778
\end{array}\right]
\end{aligned}
$$

In this matrix, a row of zero probabilities happens when the $s_{o, i}^{t, d}$ is zero. In these cases, since the probability of being in state $i$ is zero at that specific time-step, it is not possible to have probabilities of state transitions.

\section{Validation of the Inhomogeneous Markov Chain Occupancy Prediction Model}

To validate the performance of the proposed inhomogeneous Markov chain occupancy prediction model, the actual occupancy for different days of a week are compared to those resulting from the prediction model. Having the occupancy profile prediction for each occupant results in developing the occupancy rate prediction for each zone. After calculating the $P_{i j}{ }_{o}^{d}(\mathrm{t})$ matrices, the number of present occupants in each zone can be predicted. Assuming $n_{o}^{t, d}$ occupants in zone $z$, the occupancy rate of this zone for time-step $t$ of day $d$ $\left(o c c_{r z}^{t, d}\right)$ is calculated using the average number of present occupants as follows:

$$
o c c_{r z}^{t, d}=\frac{n_{o}^{t, d}}{N_{o}} \times 100 \%
$$

Unlike Equation 1, the effect of number of weeks is not shown in this equation since this parameter was already considered in the process of calculating the $P_{i j_{o}}^{d}(\mathrm{t})$ matrices. Using Equation 5, the occupancy rate at zone level is calculated and the results are used to control the lighting system. Since the prediction model demonstrated the same performance for different days of a week, Figure 8 shows the occupancy rates only for Mondays. The same comparison was made between the occupancy rates resulting from the prediction model to be used for HVAC system control (i.e., 30-minute time-step prediction), and the real data as illustrated in Figure 9.

The prediction model can accurately estimate the location of occupants at most periods of data collection during the day, which shows that the overall performance of the prediction model is satisfactory. Coefficient of determination (known as $\mathrm{R}^{2}$ ) is used as an indicator for evaluating the performance of the proposed prediction model. The values of $\mathrm{R}^{2}$ when using the proposed prediction model for the lighting control are 0.8 and 0.92 for zones 1 and 2, respectively. This would result in having 0.86 on average for this parameter $(86 \%)$ for the application of lighting system control. The same method is used to calculate the average value of $\mathrm{R}^{2}$ when using the proposed prediction model for the control of the HVAC system (68\%). These values indicate the high accuracy of the prediction model in imitating the real occupancy patterns of the open-plan office. 


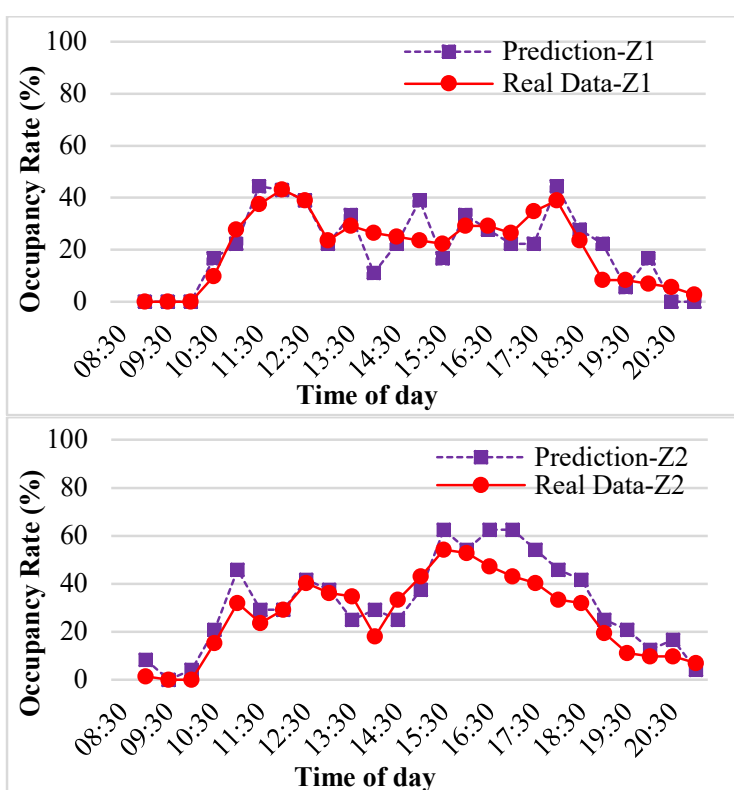

Figure 8: Comparison of predicted and the actual occupancy rates (Mondays)-Lighting control.

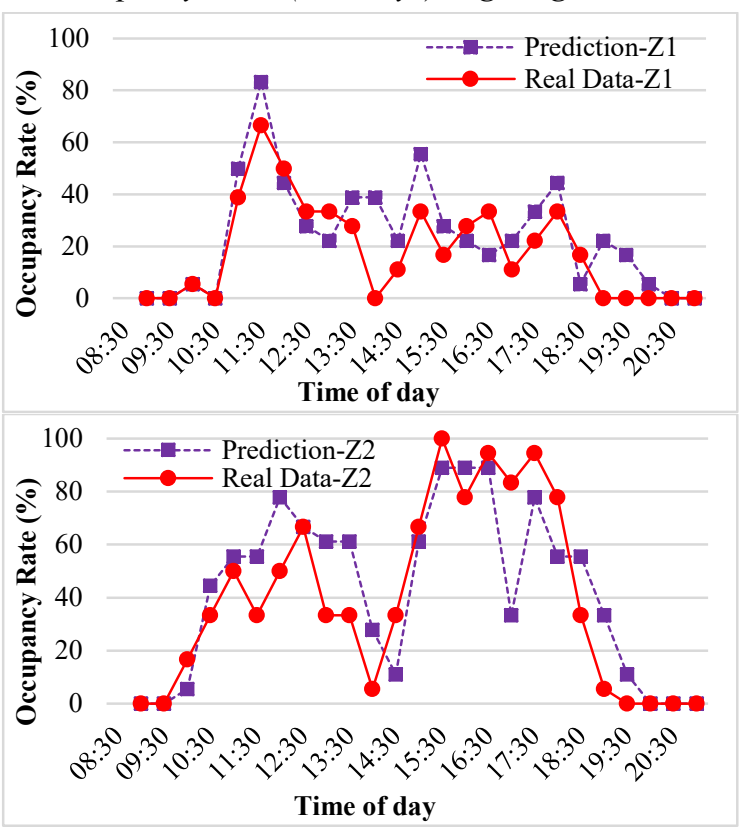

Figure 9: Comparison of predicted and the actual occupancy rates (Mondays)-HVAC control.

\section{Conclusion}

In this study, the occupancy modelling has been further enhanced using inhomogeneous Markov chain prediction model based on real occupancy patterns data. The main contributions of this research are: (1) developing a method for extracting detailed occupancy information with varying time-steps from collected RTLS occupancy data. This method can capture different resolution levels required for the application of intelligent, occupancycantered local control strategies of different building systems; (2) developing a new adaptive probabilistic occupancy prediction model based on the extracted occupancy information; and (3) developing timedependent inhomogeneous Markov chain occupancy model, which distinguishes the temporal behaviour of different occupants within an open-plan office.

Having the occupancy profile prediction for each occupant results in the occupancy rate prediction at the zone level. Analysing the changes in the occupancy $P T$ for different time horizons showed the importance of applying the effect of temporal behaviour of occupants when predicting their presence patterns. The comparison between the occupancy profiles resulting from the prediction model and the actual profiles showed that the prediction model was able to capture the actual behaviour of occupants at occupant and zone levels. The high accuracy of occupancy patterns prediction $(86 \%$ and $68 \%$ on average for the lighting and HVAC systems control, respectively) also indicates the acceptable performance of the prediction model in capturing the temporal behaviour of different occupants working in the same open-plan office.

Although the overall performance of the prediction model was satisfactory, it may not capture variations in occupancy patterns, especially in the case of open-plan offices with varying occupancy. This limitation could be solved by analysing the whole occupancy data collected over the course of a year. In addition, the effect of using other time-steps on the overall performance of the proposed model should be investigated (e.g. using finer resolution levels to control the lighting system).

There is a privacy issue when the occupants' identities are used to capture detailed occupancy patterns. However, this issue can be resolved by anonymizing the occupants' data through defining occupancy profiles per zone. In addition, having this type of data could be vital for other purposes, such as emergency and safety. Informing the monitored occupants about the benefits coming from using the real-time monitoring system for a reasonable period could also be helpful to solve this issue.

As future work, the performance of the proposed prediction model should be evaluated using occupancy data collected over a longer period of time. Using different data collection periods and smaller time-steps to control the lighting system are other parts of the future work. Future work will also use the proposed occupancy prediction model as an input to the energy simulation tools to assess different occupancy-centered local control strategies considering occupants' preferences related to building systems (e.g., HVAC and lighting systems). In addition, the proposed occupancy prediction and the energy simulation models will be encapsulated within an optimization algorithm to select the optimal settings for the building systems to achieve the objectives of minimizing energy consumption as well as the occupants' dissatisfaction. Running the simulation-based optimization model in near real-time provides more reliable occupancy responsive local control strategies to optimize building energy consumption. Thus, the ultimate results of the practical implementation of this research are a more robust operation of building systems as well as more satisfied occupants. 


\section{References}

Ai, B., Fan, Z., \& Gao, R. X. (2014). Occupancy estimation for smart buildings by an auto-regressive hidden Markov model. In American Control Conference (ACC) (pp. 2234-2239). Portland, Oregon, USA: IEEE.

Annex 53. (2016). Total Energy Use in Buildings: Analysis and Evaluation Methods. United Kingdom: AECOM Ltd.

Ardakanian, O., Bhattacharya, A., \& Culler, D. (2016). Non-intrusive techniques for establishing occupancy related energy savings in commercial buildings. Proceedings of the $3 r d$ ACM International Conference on Systems for Energy-Efficient Built Environments (pp. 21-30). Palo Alto, CA, USA: ACM.

BPIE Database. (2013). Retrieved from http://www.buildingsdata.eu/

Chang, W. K., \& Hong, T. (2013). Statistical analysis and modeling of occupancy patterns in open-plan offices using measured lighting-switch data. In Building Simulation, 6(1), 23-32.

Chen, Z., Xu, J., \& Soh, Y. C. (2015). Modeling regular occupancy in commercial buildings using stochastic models. Energy and Buildings, 103, 216-223.

Douc, R., Moulines, E., \& Rosenthal, J. S. (2004). Quantitative bounds on convergence of timeinhomogeneous Markov chains. Annals of Applied Probability, 14(4), 1643-1665.

Fauzi, C., \& Sulistyo, S. (2018). The Model of HVAC's Control Technique Based on Occupancy in Smart Building for Energy Saving. 2018 4th International Conference on Science and Technology (ICST) (pp. 16). Yogyakarta, Indonesia: IEEE.

Grizzly Analytics. (2015). Seeing Quuppa's indoor location technology at MWC 2015.

Haldi, F., \& Robinson, D. (2008). On the behaviour and adaptation of office occupants. Building and environment, 43(12), 2163-2177.

Han, Z., Gao, R. X., \& Fan, Z. (2012). Occupancy and indoor environment quality sensing for smart buildings. In Instrumentation and Measurement Technology Conference (I2MTC), 2012 IEEE International (pp. 882-887). IEEE.

Hong, T., D'Oca, S., Turner, W. J., \& Taylor-Lange, S. C. (2015). An ontology to represent energy-related occupant behavior in buildings. Part I: Introduction to the DNAs framework. Building and Environment, 92, 764-777.

Li, N., Calis, G., \& Becerik-Gerber, B. (2012). Measuring and monitoring occupancy with an RFID based system for demand-driven HVAC operations. Automation in construction, 24, 89-99.

Liu, Z. (2017). Simulation of Local Climate Control in Shared Offices Based on Occupants Locations and Preferences. Montreal, Canada: A thesis in the Department of Building, Civil, and Environmental Engineering, Concordia University.

Natural Resources Canada. (2015). Survey of Commercial and Institutional Energy Use (SCIEU) - Buildings 2009. Retrieved from Natural Resources Canada: https://oee.nrcan.gc.ca/corporate/statistics/neud/dpa/d ata_e/databases.cfm

Nguyen, T. A., \& Aiello, M. (2013). Energy intelligent buildings based on user activity: A Survey. Energy and buildings, 56, 244-257.

Quuppa. (2017). One for all. Retrieved from Quuppa Do More With Location: http:/quuppa.com/applications/

Richardson, I., Thomson, M., \& Infield, D. (2008). A high-resolution domestic building occupancy model for energy demand simulations. Energy and buildings, 40(8), 1560-1566.

Salimi, S., \& Hammad, A. (2018). Critical Review and Research Roadmap of Office Building Energy Management Based on Occupancy Monitoring. Energy and Buildings. doi:10.1016/j.enbuild.2018.10.007

US Energy Information Administration (EIA). (2013). Annual Energy Outlook, DOE/EIA. Regional analysis of building distributed energy costs and CO2 Abatement: a U.S.-China comparison.

Virote, J., \& Neves-Silva, R. (2012). Stochastic models for building energy prediction based on occupant behavior assessment. Energy and Buildings, 53, 183193.

Wang, W., Lin, Z., \& Chen, J. (2017). Promoting Energy Efficiency of HVAC Operation in Large Office Spaces with a Wi-Fi Probe enabled Markov Time Window Occupancy Detection Approach. Energy Procedia, 143, 204-209.

Wei, S., Yong, J., Ng, B., Tindall, J., Lu, Q., \& Du, H. (2018). Occupant Adaptive Behaviour: an Effective Method towards Energy Efficient Buildings. CIBSE Technical Symposium. London, UK.

Yamaguchi, Y., Shimoda, Y., \& Mizuno, M. (2003). Development of district energy system simulation model based on detailed energy demand model. In Proceeding of Eighth International IBPSA Conference, (pp. 1443-1450). Eindhoven, Netherlands. 